\title{
Multiplicity of Solutions for an Elliptic Problem with Critical Sobolev-Hardy Exponents and Concave-Convex Nonlinearities
}

\author{
Juan $\mathrm{Li}^{1}$ and Yuxia Tong ${ }^{2}$ \\ ${ }^{1}$ Department of Mathematics, Ningbo University, Ningbo 315211, China \\ ${ }^{2}$ College of Sciences, Hebei United University, Tangshan 063009, China \\ Correspondence should be addressed to Juan Li; juanjuan-li@163.com
}

Received 29 September 2013; Accepted 25 January 2014; Published 6 March 2014

Academic Editor: Shusen Ding

Copyright (C) $2014 \mathrm{~J}$. Li and Y. Tong. This is an open access article distributed under the Creative Commons Attribution License, which permits unrestricted use, distribution, and reproduction in any medium, provided the original work is properly cited.

We study the existence of multiple solutions for the following elliptic problem: $-\Delta_{p} u-\mu\left(|u|^{p-2} u /|x|^{p}\right)=\left(|u|^{p^{*}(t)-2} /|x|^{t}\right) u+$ $\lambda\left(|u|^{q-2} /|x|^{s}\right) u, u \in W_{0}^{1, p}(\Omega)$. We prove that if $1 \leq q<p<N$, then there is a $\mu_{0}$, such that for any $\mu \in\left(0, \mu_{0}\right)$, the above mentioned problem possesses infinitely many weak solutions. Our result generalizes a similar result (Azorero and Alonso, 1991).

\section{Introduction and Main Results}

In this paper, we study the existence of multiple solutions to the following elliptic problem:

$$
\begin{gathered}
-\Delta_{p} u-\mu \frac{|u|^{p-2} u}{|x|^{p}}=\frac{|u|^{p^{*}(t)-2}}{|x|^{t}} u+\lambda \frac{|u|^{q-2}}{|x|^{s}} u, \quad x \in \Omega, \\
u(x)=0, \quad x \in \partial \Omega,
\end{gathered}
$$

where $\Omega \subset \mathfrak{R}^{N}(N \geq 3)$ is a smooth bounded domain containing the origin $0, \Delta_{p} u=\operatorname{div}\left(|\nabla u|^{p-2} \nabla u\right)$ is the pLaplacian of $u, 1<p<N, 0 \leq \mu<\bar{\mu} \equiv((N-p) / p)^{p}, 0 \leq t$, $s<p, 1 \leq q<p$, and $p^{*}(t)=p(N-t) /(N-p)$ is the critical Sobolev-Hardy exponent; note that $p^{*}(0)=p^{*} \equiv p N /(N-p)$ is the critical Sobolev exponent.

Problem (1) is related to the well-known Sobolev-Hardy inequalities [1]:

$$
\begin{array}{r}
\left(\int_{\mathfrak{R}^{N}} \frac{|u|^{p^{*}(t)}}{|x|^{t}} d x\right)^{p / p^{*}(t)} \leq C \int_{\mathfrak{R}^{N}}|\nabla u|^{p} d x, \\
\forall u \in W_{0}^{1, p}(\Omega) .
\end{array}
$$

As $t=p, p^{*}(t)=p$, then the well-known Hardy inequality holds $[1,2]$ :

$$
\int_{\mathfrak{R}^{N}} \frac{|u|^{p}}{|x|^{p}} d x \leq \frac{1}{\bar{\mu}} \int_{\mathfrak{R}^{N}}|\nabla u|^{p} d x, \quad \forall u \in W_{0}^{1, p}(\Omega) .
$$

In this paper, we use $L^{q}\left(\Omega,|x|^{s}\right)$ to denote the usual weighted $L^{q}(\Omega)$ space with the weight $|x|^{s}$. In $W_{0}^{1, p}(\Omega)$, for $\mu \in[0, \bar{\mu})$, we use the norm

$$
\|u\|=\|u\|_{W_{0}^{1, p}(\Omega)}=\left(\int_{\Omega}\left(|\nabla u|^{p}-\mu \frac{|u|^{p}}{|x|^{p}}\right) d x\right)^{1 / p} .
$$

By (3), this norm is equivalent to the usual norm $\left(\int_{\Omega}|\nabla u|^{p} d x\right)^{1 / p}$. By the Hardy inequality and the SobolevHardy inequality, for $0 \leq \mu<\bar{\mu}, 0 \leq t<p$, we can define the following best constants:

$$
A_{\mu, t}(\Omega)=\inf _{u \in W_{0}^{1, p}(\Omega) \backslash\{0\}} \frac{\|u\|^{p}}{\left(\int_{\Omega}\left(|u|^{p^{*}(t)} /|x|^{t}\right) d x\right)^{p / p^{*}(t)}} .
$$

Note that $A_{\mu, 0}$ is the best constant in the Sobolev inequality, that is,

$$
A_{\mu, 0}(\Omega)=\inf _{u \in W_{0}^{1, p}(\Omega) \backslash\{0\}} \frac{\int_{\Omega}\left(|\nabla u|^{p}-\mu\left(|u|^{p} /|x|^{p}\right)\right) d x}{\left(\int_{\Omega}|u|^{p^{*}} d x\right)^{p / p^{*}}} .
$$


The energy functional of (1) is defined as follows:

$$
\begin{aligned}
I(u)= & \frac{1}{p} \int_{\Omega}\left(|\nabla u|^{p}-\mu \frac{|u|^{p}}{|x|^{p}}\right) d x-\frac{1}{p^{*}(t)} \int_{\Omega} \frac{|u|^{p^{*}(t)}}{|x|^{t}} d x \\
& -\frac{\lambda}{q} \int_{\Omega} \frac{|u|^{q}}{|x|^{s}} d x .
\end{aligned}
$$

Then, $I(u)$ is well defined on $W_{0}^{1, p}(\Omega)$ and belongs to $C^{1}\left(W_{0}^{1, p}(\Omega), \Re\right)$. The solutions of problem (1) are then the critical points of the functional $I$.

In recent years, the quasilinear problems related to Hardy inequality and Sobolev-Hardy inequality have been studied by some authors [3-7]. Ghoussoub and Yuan [5] studied problem (1) with $\mu=0, s=0$, and $p \leq q \leq p^{*}$ and proved the existence results of positive solutions and sign-changing solutions. Kang in $[3,4]$ studied $(1,1)$ when $p \leq q \leq p^{*}(s)$ and verified the existence of positive solutions of (1) when the parameters $p, q, s, \lambda, \mu$ satisfy suitable conditions. To the best of our knowledge, there are few results of problem (1) involving the p-sublinear of $1 \leq q<p<N$. We are only aware of the works [6-9] which studied the existence and multiplicity of solution of problem (1) involving weight functions. Azorero and Alonso [9] studied problem (1) with $s=t=\mu=0,1<q<p$ and proved that there exists $\lambda_{1}$, such that (1) has infinitely many solutions for $\lambda \in\left(0, \lambda_{1}\right)$. Hsu [7] studied problem (1) and proved that there exists $\Lambda_{0}>0$ such that (1) has at least two positive solutions for $\lambda \in\left(0, \Lambda_{0}\right)$. In this paper, we study (1) and extend the results of $[7,9]$.

Throughout this paper, let $R_{0}$ be the positive constant such that $\Omega \subset B\left(0, R_{0}\right)$, where $B\left(0, R_{0}\right)=\left\{x \in \Re^{N}:|x|<\right.$ $\left.R_{0}\right\}$. By Holder inequalities, for all $u \in W_{0}^{1, p}(\Omega)$, we obtain

$$
\begin{aligned}
\int_{\Omega} \frac{|u|^{q}}{|x|^{s}} d x & \\
\leq & \left(\int_{B\left(0, R_{0}\right)}|x|^{\left(t q-s p^{*}(t)\right) /\left(p^{*}(t)-q\right)} d x\right)^{\left(p^{*}(t)-q\right) / p^{*}(t)} \\
& \times\left(\int_{\Omega} \frac{|u|^{p^{*}(t)}}{|x|^{t}} d x\right)^{q / p^{*}(t)} \\
\leq & \left(N \omega_{N} \int_{0}^{R_{0}} r^{\left(\left(t q-s p^{*}(t)\right) /\left(p^{*}(t)-q\right)\right)+N-1} d r\right)^{\left(p^{*}(t)-q\right) / p^{*}(t)} \\
& \times\left(\int_{\Omega} \frac{|u|^{p^{*}(t)}}{|x|^{t}} d x\right)^{q / p^{*}(t)} \\
= & \left(\frac{N \omega_{N} R_{0}^{\left(\left(t q-s p^{*}(t)\right) /\left(p^{*}(t)-q\right)\right)+N}\left(p^{*}(t)-q\right)}{q(t-N)+p^{*}(t)(N-s)}\right)^{\left(p^{*}(t)-q\right) / p^{*}(t)}
\end{aligned}
$$

$$
\begin{gathered}
\times\left(\int_{\Omega} \frac{|u|^{p^{*}(t)}}{|x|^{t}} d x\right)^{q / p^{*}(t)} \\
=c_{0}\left(\int_{\Omega} \frac{|u|^{p^{*}(t)}}{|x|^{t}} d x\right)^{q / p^{*}(t)},
\end{gathered}
$$

where $\omega_{N}=2 \pi^{N / 2} / N \Gamma(N / 2)$ is the volume of the unit ball in $\mathfrak{R}^{N}$. The following inequality comes from the paper [7]:

$$
\int_{\Omega} \frac{|u|^{q}}{|x|^{s}} d x \leq\left(\frac{N \omega_{N} R_{0}^{N-s}}{N-s}\right)^{\left(p^{*}(s)-q\right) / p^{*}(s)} A_{\mu, s}^{-q / p}\|u\|^{q} .
$$

Now we are ready to state our main results.

Theorem 1. If $\Omega \subset \mathfrak{R}^{N}$ is a bounded domain in $\mathfrak{R}^{N}$, and $1 \leq q<p<N$, then there is a $\mu_{0}>0$ such that problem (1) possesses infinitely many weak solutions in $W_{0}^{1, p}(\Omega)$ for any $\mu \in\left(0, \mu_{0}\right)$.

\section{The Palais-Smale Condition}

Let $X$ be a Banach space and $X^{-1}$ be the dual space of $X$. The functional $I \in C^{1}(X, \Re)$ is said to satisfy the PalaisSmale condition at level $c\left((P S)_{c}\right)$, if any sequence $\left(u_{n}\right) \subset X$ satisfying

$$
I\left(u_{n}\right) \longrightarrow c, \quad I^{\prime}\left(u_{n}\right) \longrightarrow 0 \quad \text { strongly in } X^{-1} \text { as } n \rightarrow \infty
$$

contains a subsequence converging in $X$ to a critical point of the functional $I$. In this paper, we will take $X=W_{0}^{1, p}(\Omega)$.

Lemma 2. Let $\left\{u_{n}\right\} \subset W_{0}^{1, p}(\Omega)$ be a Palais-Smale sequence for I defined by (7), that is,

$$
\begin{gathered}
I\left(u_{n}\right) \longrightarrow c, \\
I^{\prime}\left(u_{n}\right) \longrightarrow 0 \quad \text { in } W^{-1, p^{\prime}}(\Omega), \quad \frac{1}{p}+\frac{1}{p^{\prime}}=1 .
\end{gathered}
$$

If $1 \leq q<p$ and $c<(p-t) / p(N-t)\left(A_{\mu, t}\right)^{(N-t) /(p-t)}-$ $K \lambda^{p^{*}(t) /\left(p^{*}(t)-q\right)}$, and $K$ depends on $p, q, N$, then, there exists a subsequence $\left\{u_{n_{k}}\right\} \subset\left\{u_{n}\right\}$, strongly convergent in $W_{0}^{1, p}(\Omega)$.

Proof. By (11) and (12), it is easy to prove that the sequence $u_{n}$ is bounded in $W_{0}^{1, p}(\Omega)$. Passing to a subsequence if necessary, we may assume that, as $n \rightarrow \infty$,

$$
\begin{gathered}
u_{n} \rightarrow u \quad \text { weakly in } W_{0}^{1, p}(\Omega), \\
u_{n} \rightarrow u \quad \text { weakly in } L^{p^{*}(t)}\left(\Omega,|x|^{-t}\right), \\
u_{n} \rightarrow u \quad \text { weakly in } L^{p}\left(\Omega,|x|^{-p}\right), \\
u_{n} \longrightarrow u \quad \text { strongly in } L^{q}\left(\Omega,|x|^{-s}\right), \\
u_{n} \longrightarrow u \quad \text { strongly in } L^{p}(\Omega), \\
u_{n} \longrightarrow u \quad \text { a.e. in } \Omega .
\end{gathered}
$$


Then, $u \in W_{0}^{1, p}(\Omega)$ is a solution of problem (1). By the concentration compactness principle (see $[10,11]$ ), there exists a subsequence, still denoted by $u_{n}$, at the most countable set $j$, a set of different points $\left\{x_{j}\right\}_{j \in j} \subset \Omega \backslash\{0\}$, sets of nonnegative real numbers $\left\{\widetilde{\mu}_{j}\right\}_{j \in j \cup\{0\}},\left\{\widetilde{v}_{j}\right\}_{j \in j \cup\{0\}}$, and nonnegative real numbers $\tilde{\tau}_{0}$ and $\widetilde{\gamma}_{0}$, such that

$$
\begin{gathered}
\left|\nabla u_{n}\right|^{p}-d \widetilde{\mu} \geq|\nabla u|^{p}+\sum_{j \in j} \widetilde{\mu}_{j} \delta_{x_{j}}+\widetilde{\mu}_{0} \delta_{0}, \\
\left|u_{n}\right|^{p^{*}}-d \widetilde{v}=|u|^{p^{*}}+\sum_{j \in j} \widetilde{v}_{j} \delta_{x_{j}}+\widetilde{v}_{0} \delta_{0}, \\
\frac{\left|u_{n}\right|^{p^{*}(t)}}{|x|^{t}} \rightarrow d \widetilde{\tau}=\frac{|u|^{p^{*}(t)}}{|x|^{t}}+\widetilde{\tau}_{0} \delta_{0}, \\
\frac{\left|u_{n}\right|^{p}}{|x|^{p}}-d \widetilde{\gamma}=\frac{|u|^{p}}{|x|^{p}}+\widetilde{\gamma}_{0} \delta_{0},
\end{gathered}
$$

where $\delta_{x}$ is the Dirac mass at $x$.

Case $1\left(t=s=0\right.$ and $\left.p^{*}(t)=p^{*}\right)$. We claim that $\rho$ is finite, and, for any $j \in j$, either

$$
\widetilde{v}_{j}=0 \quad \text { or } \quad \widetilde{v}_{j} \geq\left(A_{\mu, 0}\right)^{N / p}
$$

In fact, let $\varepsilon>0$ be small enough such that $0 \bar{\epsilon} B\left(x_{j}, \varepsilon\right)$ and $B\left(x_{i}, \varepsilon\right) \bigcap B\left(x_{j}, \varepsilon\right)=\emptyset$ for $i \neq j, i, j \in \jmath$. We consider $\varphi_{j} \in$ $C_{0}^{\infty}\left(\Re^{N}\right)$, such that

$$
\begin{gathered}
\varphi_{j} \equiv 1 \quad \text { on } B\left(x_{j}, \frac{\varepsilon}{2}\right), \\
\varphi_{j} \equiv 0 \quad \text { on } B\left(x_{j}, \varepsilon\right)^{c}, \\
\left|\nabla \varphi^{j}\right| \leq \frac{4}{\varepsilon}, \\
0 \leq \varphi_{j} \leq 1 .
\end{gathered}
$$

It is clear that the sequence $\left\{\varphi_{j} u_{n}\right\}$ is bounded in $W_{0}^{1, p}(\Omega)$. Note that

$$
\begin{aligned}
\left\langle I^{\prime}\left(u_{n}\right), u_{n} \varphi_{j}\right\rangle= & \int_{\Omega}\left|\nabla u_{n}\right|^{p} \varphi_{j} d x \\
& +\int_{\Omega} u_{n}\left|\nabla u_{n}\right|^{p-2} \nabla u_{n} \nabla \varphi_{j} d x \\
& -\mu \int_{\Omega} \frac{\left|u_{n}\right|^{p}}{|x|^{p}} \varphi_{j} d x \\
& -\int_{\Omega}\left|u_{n}\right|^{p^{*}} \varphi_{j} d x-\lambda \int_{\Omega}\left|u_{n}\right|^{q} \varphi_{j} d x .
\end{aligned}
$$

By (13), (16), and the Holder inequality, we obtain

$$
\begin{aligned}
0 & \leq\left.\lim _{\varepsilon \rightarrow 0} \lim _{n \rightarrow \infty}\left|\int_{\Omega} u_{n}\right| \nabla u_{n}\right|^{p-2} \nabla u_{n} \nabla \varphi_{j} d x \mid \\
& \leq \operatorname{Cim}_{\varepsilon \rightarrow 0}\left(\int_{B\left(x_{j}, \varepsilon\right)}|u|^{p^{*}} d x\right)^{1 / p^{*}}=0, \\
& \lim _{\varepsilon \rightarrow 0} \lim _{n \rightarrow \infty}\left|\int_{\Omega} \frac{\left|u_{n}\right|^{p}}{|x|^{p}} \varphi_{j} d x\right| \\
& \leq \lim _{\varepsilon \rightarrow 0} \lim _{n \rightarrow \infty}\left|\int_{B_{\varepsilon}\left(x_{j}\right)} \frac{\left|u_{n}\right|^{p}}{\left(\left|x_{j}\right|-\varepsilon\right)^{p}} \varphi_{j} d x\right|=0 .
\end{aligned}
$$

From (12) (18), we get that

$$
0=\lim _{\varepsilon \rightarrow 0} \lim _{n \rightarrow \infty}\left\langle I^{\prime}\left(u_{n}\right), u_{n} \varphi_{j}\right\rangle \geq \tilde{\mu}_{j}-\widetilde{v}_{j} .
$$

By the Sobolev inequality, $A_{0,0} v_{j}^{p / p^{*}} \leq \widetilde{\mu}_{j}$, hence, we deduce that

$$
\widetilde{v}_{j}=0 \quad \text { or } \quad \tilde{v}_{j} \geq\left(A_{0,0}\right)^{N / p},
$$

which implies that $\jmath$ is finite.

Now we consider the possibility of concentration at the origin. Let $\varepsilon>0$ be small enough such that $x_{j} \bar{\epsilon} B(0, \varepsilon), \forall j \in \jmath$. Take $\varphi^{0} \in C_{0}^{\infty}\left(\Re^{N}\right)$ such that

$$
\begin{gathered}
\varphi^{0} \equiv 1 \quad \text { on } B\left(0, \frac{\varepsilon}{2}\right), \\
\varphi^{0} \equiv 0 \quad \text { on } B(0, \varepsilon)^{c}, \\
\left|\nabla \varphi^{0}\right| \leq \frac{4}{\varepsilon} .
\end{gathered}
$$

By (13) and (14), we also get that

$$
\begin{aligned}
& 0=\lim _{\varepsilon \rightarrow 0} \lim _{n \rightarrow \infty}\left\langle I^{\prime}\left(u_{n}\right), u_{n} \varphi^{0}\right\rangle \\
& =\lim _{\varepsilon \rightarrow 0}\left(\int_{\Omega} \varphi^{0} d \widetilde{\mu}-\int_{\Omega} \varphi^{0} d \widetilde{\gamma}-\int_{\Omega} \varphi^{0} d \widetilde{\nu}\right. \\
& \left.\quad-\lambda \int_{\Omega} \varphi^{0} \frac{|u|^{q}}{|x|^{s}} d x\right) \\
& \geq \widetilde{\mu}_{0}-\mu \widetilde{\gamma}_{0}-\widetilde{\nu}_{0} .
\end{aligned}
$$

By the definition of $A_{\mu, 0}$, we deduce that

$$
A_{\mu, 0} \widetilde{\nu}_{0}^{p / p^{*}} \leq \widetilde{\mu}_{0}-\mu \widetilde{\gamma}_{0} .
$$

From (22) we have

$$
A_{\mu, 0} \widetilde{v}_{0}^{p / p^{*}} \leq \widetilde{\mu}_{0}-\mu \widetilde{\gamma}_{0} \leq \widetilde{\nu}_{0},
$$

which implies that $\widetilde{v}_{0}=0$ or

$$
\widetilde{v}_{0} \geq\left(A_{\mu, 0}\right)^{N / p} .
$$


We will prove that $(25)$ and $\widetilde{v}_{j} \geq\left(A_{0,0}\right)^{N / p}$ are not possible. By (13) and (14),

$$
\begin{aligned}
c= & \lim _{n \rightarrow \infty} I\left(u_{n}\right)=\lim _{n \rightarrow \infty}\left\{I\left(u_{n}\right)-\frac{1}{p}\left\langle I^{\prime}\left(u_{n}\right), u_{n}\right\rangle\right\} \\
= & \lim _{n \rightarrow \infty}\left\{\frac{1}{N} \int_{\Omega}\left|u_{n}\right|^{p^{*}} d x+\lambda\left(\frac{1}{p}-\frac{1}{q}\right) \int_{\Omega}\left|u_{n}\right|^{q} d x\right\} \\
\geq & \frac{1}{N}\left(\int_{\Omega}|u|^{p^{*}} d x+\sum_{j \in j} \widetilde{v}_{j}+\widetilde{v}_{0}\right)+\lambda\left(\frac{1}{p}-\frac{1}{q}\right) \int_{\Omega}|u|^{q} d x \\
\geq & \frac{1}{N} \int_{\Omega}|u|^{p^{*}} d x+\frac{1}{N} \min \left\{\left(A_{\mu, 0}\right)^{N / p},\left(A_{0,0}\right)^{N / p}\right\} \\
& +\lambda\left(\frac{1}{p}-\frac{1}{q}\right) \int_{\Omega}|u|^{q} d x \\
\geq & \frac{1}{N} \int_{\Omega}|u|^{p^{*}} d x+\frac{1}{N}\left(A_{\mu, 0}\right)^{N / p} \\
& +\lambda\left(\frac{1}{p}-\frac{1}{q}\right) \int_{\Omega}|u|^{q} d x .
\end{aligned}
$$

By applying the Holder inequality at (26), we have

$$
\begin{aligned}
c \geq & \frac{1}{N}\left(A_{\mu, 0}\right)^{N / p}+\frac{1}{N} \int_{\Omega}|u|^{p^{*}} d x \\
& -\lambda\left(\frac{1}{q}-\frac{1}{p}\right)|\Omega|^{\left(p^{*}-q\right) / p^{*}}\left(\int_{\Omega}|u|^{p^{*}} d x\right)^{q / p^{*}} .
\end{aligned}
$$

Let $f_{1}(x)=c_{1} x^{p^{*}}-\lambda c_{2} x^{q}, c_{1}=1 / N, c_{2}=(1 / q)-(1 / p)$. This function obtains its absolute minimum (for $x>0$ ) at point $x_{0}=\left(\lambda c_{2} q / p^{*} c_{1}\right)^{1 /\left(p^{*}-q\right)}$. That is,

$$
f_{1}(x) \geq f_{1}\left(x_{0}\right)=-K_{1} \lambda^{p^{*} /\left(p^{*}-q\right)},
$$

where

$$
\begin{aligned}
K_{1}= & c_{2}^{p /\left(p^{*}-q\right)} c_{1}^{-q /\left(p^{*}-q\right)} \\
& \times\left(\left(\frac{q}{p^{*}}\right)^{q /\left(p^{*}-q\right)}-\left(\frac{q}{p^{*}}\right)^{p^{*} /\left(p^{*}-q\right)}\right)>0,
\end{aligned}
$$

because of $1<q<p<N p /(N-p)$. But this result contradicts the hypothesis. Then, $\widetilde{v}_{j}=0 \forall j \in j \cup\{0\}$ and we conclude.

Case $2\left(0<t<p\right.$, then $\left.p<p^{*}(t)<p^{*}\right)$. We only need to consider the possibility of concentration at the origin. Let $\varepsilon>0$ be small enough such that $B(0, \varepsilon) \subset \Omega$. Take $\varphi^{0}$ a smooth cut-off function centered at the origin such that $0 \leq \varphi^{0} \leq 1$, $\varphi^{0}=1$ for $|x| \leq \varepsilon / 2, \varphi^{0}=0$ for $|x| \geq \varepsilon$, and $\left|\nabla \varphi^{0}\right| \leq 4 / \varepsilon$. By (13) and (14), we get that

$$
\begin{aligned}
0 & =\lim _{\varepsilon \rightarrow 0} \lim _{n \rightarrow \infty}\left\langle I^{\prime}\left(u_{n}\right), u_{n} \varphi^{0}\right\rangle \\
& =\lim _{\varepsilon \rightarrow 0}\left(\int_{\Omega} \varphi^{0} d \widetilde{\mu}-\mu \int_{\Omega} \varphi^{0} d \widetilde{\gamma}-\int_{\Omega} \varphi^{0} d \widetilde{\tau}-\lambda \int_{\Omega} \varphi^{0} \frac{|u|^{q}}{|x|^{s}} d x\right) \\
& \geq \widetilde{\mu}_{0}-\mu \widetilde{\gamma}_{0}-\widetilde{\tau}_{0} .
\end{aligned}
$$

By the definition of $A_{\mu, t}$, we deduce that

$$
A_{\mu, t} \widetilde{\tau}_{0}^{p / p^{*}(t)} \leq \widetilde{\mu}_{0}-\mu \widetilde{\gamma}_{0}
$$

From (30), we have

$$
A_{\mu, t} \widetilde{\tau}_{0}^{p / p^{*}(t)} \leq \widetilde{\tau}_{0}
$$

which implies that $\widetilde{\tau}_{0}=0$ or

$$
\widetilde{\tau}_{0} \geq\left(A_{\mu, t}\right)^{(N-t) /(p-t)} .
$$

We will prove (33) is not possible. From the above arguments and (8), we conclude that

$$
\begin{aligned}
c= & \lim _{n \rightarrow \infty} J\left(u_{n}\right)=\lim _{n \rightarrow \infty}\left\{J\left(u_{n}\right)-\frac{1}{p}\left\langle J\left(u_{n}\right), u_{n}\right\rangle\right\} \\
\geq & \frac{p-t}{p(N-t)} \int_{\Omega} \frac{|u|^{p^{*}(t)}}{|x|^{t}} d x+\frac{p-t}{p(N-t)}\left(A_{\mu, t}\right)^{(N-t) /(p-t)} \\
& +\lambda c_{0}\left(\frac{1}{q}-\frac{1}{p}\right)\left(\int_{\Omega} \frac{|u|^{p^{*}(t)}}{|x|^{t}} d x\right)^{q / p^{*}(t)} .
\end{aligned}
$$

Let $f_{2}(x)=c_{3} x^{p^{*}(t)}-\lambda c_{4} x^{q}, c_{3}=(p-t) / p(N-t), c_{4}=$ $c_{0}((1 / q)-(1 / p))$. This function obtains its absolute minimum at point $x_{0}=\left(\lambda c_{4} q / p^{*}(t) c_{3}\right)^{1 /\left(p^{*}-q\right)}$. That is,

$$
f_{2}(x) \geq f_{2}\left(x_{0}\right)=-K_{2} \lambda^{p^{*}(t) /\left(p^{*}(t)-q\right)} .
$$

But this result contradicts the hypothesis. Hence, up to a subsequence, we obtain that $u_{n} \rightarrow u$ strongly in $W_{0}^{1, p}(\Omega)$.

Thus, the proof of the Lemma is completed.

\section{Existence of Infinitely Many Solutions}

In this section, we will prove our main result of Theorem 1. We first recall some concepts and results in minimax theory.

Let $X$ be a Banach space, and $\Sigma$ denote all closed subsets of $X-\{0\}$ which are symmetric with respect to the origin. For $A \in \Sigma$, we define the genus $\gamma(A)$ by

$$
\begin{aligned}
& \gamma(A) \\
& =\min \left\{k \in \mathbf{N}: \exists \phi \in C\left(A ; \mathbf{R}^{k} \backslash\{0\}\right), \phi(x)=-\phi(-x)\right\},
\end{aligned}
$$

if the minimum exists, and if such a minimum does not exist, then we define $\gamma(A)=\infty$. The main properties of the genus are contained in the following lemma (see [12] for the details). 
Lemma 3. Let $A, B \in \Sigma$. Then one has the following.

(1) If there exists $f \in C(A, B)$, odd, then $\gamma(A) \leq(B)$.

(2) If $A \subset B$, then $\gamma(A) \leq \gamma(B)$.

(3) If there exists an odd homeomorphism between $A$ and $B$, then $\gamma(A)=\gamma(B)$.

(4) If $S^{N-1}$ is the sphere in $\mathfrak{R}^{N}$, then $\gamma\left(S^{N-1}\right)=N$.

(5) Consider $\gamma(A \cup B) \leq \gamma(A)+\gamma(B)$.

(6) If $\gamma(B)<+\infty$, then $\gamma(\overline{A-B}) \geq \gamma(A)-\gamma(B)$.

(7) If $A$ is compact, then $\gamma(A)<+\infty$, and there exists $\delta>$ 0 such that $\gamma\left(N_{\delta}(A)\right)=\gamma(A)$, where $N_{\delta}(A)=\{x \in X$ : $d(x, A) \leq \delta\}$.

(8) If $X_{0}$ is a subspace of $X$ with codimension $k$, and $\gamma(A)<$ $k$, then $A \cap X_{0} \neq \emptyset$.

Let $X$ be a Banach space and $E$ be a $C^{1}$ functional on $X$. Denote $E^{c}=\{u \in X \mid E(u) \leq c\}, \Sigma_{k}=\left\{C \subset W_{0}^{1, p}(\Omega)-\right.$ $\{0\}, C$ is closed, $C=-C, \gamma(C) \geq k\}$.

Given the functional $I$, under the hypothesis $1<q<p<$ $n$, using Sobolev's equality and (9), we obtain

$$
I(u) \geq \frac{1}{p}\|u\|^{p}-\frac{1}{p^{*}(t) A_{\mu, t}^{p^{*}(t) / p}}\|u\|^{p^{*}(t)}-\frac{\lambda c_{0}}{q}\|u\|^{q} .
$$

If we define for $x \geq 0$

$$
h(x)=\frac{1}{p} x^{p}-\frac{1}{p^{*}(t) A_{\mu, t}^{p^{*}(t) / p}} x^{p^{*}(t)}-\frac{\lambda c_{0}}{q} x^{q},
$$

then

$$
I(u) \geq h(\|u\|)
$$

Because $p^{*}(t)>p$ and $h(x) \rightarrow-\infty$, as $x \rightarrow+\infty$, it is easy to see that there exists $0<\mu_{0} \leq 1$ such that, if $0<\mu \leq \mu_{0}, h$ attains its positive maximum.

From the structure of $h(x)$, we see that there are constants $0<R_{0}<R_{1}$, such that $h\left(R_{0}\right)=h\left(R_{1}\right)=0, h(R) \leq 0$ if $R<R_{0}$, $h(R)>0$ if $R_{0}<R<R_{1}$, and $h(R)<0$ if $R>R_{1}$. Following [9], let $\tau: \mathbf{R}^{+} \rightarrow[0,1] \in C^{\infty}$ be nonincreasing, such that

$$
\tau(x)= \begin{cases}1, & \text { if } 0 \leq x \leq R_{0}, \\ 0, & \text { if } x \geq R_{1}\end{cases}
$$

and let $\varphi(u)=\tau(\|u\|)$; we consider the truncated functional

$$
\begin{aligned}
J(u)= & \frac{1}{p} \int_{\Omega}\left(|\nabla u|^{p}-\mu \frac{u^{p}}{|x|^{p}}\right) d x \\
& -\frac{1}{p^{*}(t)} \int_{\Omega} \frac{|u|^{p^{*}(t)} \varphi(u)}{|x|^{t}} d x-\frac{\lambda}{q} \int_{\Omega} \frac{|u|^{q}}{|x|^{s}} d x .
\end{aligned}
$$

Similar to (39), we have $J(u) \geq \bar{h}(\|u\|)$, where

$$
\bar{h}(x)=\frac{1}{p} x^{p}-\frac{\tau(x)}{p^{*}(t) A_{\mu, t}^{p^{*}(t) / p}} x^{p^{*}(t)}-\frac{\lambda c_{0}}{q} x^{q} .
$$

Clearly, $\bar{h}(x) \geq h(x)$ for $x \geq 0$ and $\bar{h}(x)=h(x)$ if $0 \leq x \leq R_{0}$, $\bar{h}(x) \geq h(x) \geq 0$, if $R_{0}<R<R_{1}$, and if $x>R_{1}, \bar{h}(x)=$ $(1 / p) x^{p}-\left(\lambda c_{0} / q\right) x^{q}$ is strictly increasing, and so $\bar{h}(x)>0$, if $x>R_{1}$. Consequently, $\bar{h}(x) \geq 0$ for $x \geq R_{0}$.

Lemma 4. (1) Consider $J \in C^{1}\left(W_{0}^{1, p}(\Omega), \mathbf{R}\right)$.

(2) If $J(u) \leq 0$, then $\|u\| \leq R_{0}$ and $I(v)=J(v)$ for all $v$ in $a$ small enough neighborhood of $u$.

(3) There exists $\lambda_{0}>0$, such that if $0<\lambda<\lambda_{0}$, then $J$ verifies a local Palais-Smale condition for $c \leq 0$.

Proof. (1) and (2) are immediate. To prove (3), observe that all Palais-Smale sequences for $J$ with $c \leq 0$ must be bounded; then, by Lemma 2 , if $\lambda$ verifies $((p-t) / p(N-$ $t))\left(A_{\mu, t}\right)^{(N-t) /(p-t)}-K \lambda^{p^{*}(t) /\left(p^{*}(t)-q\right)} \geq 0$, there exists a convergent subsequence.

Now, we use the idea in [9] to construct negative critical values of $J$ via genus.

Lemma 5. Given $n \in N$, there is an $\varepsilon(n)>0$, such that

$$
\gamma\left(\left\{u \in W_{0}^{1, p}(\Omega): J(u) \leq-\varepsilon(n)\right\}\right) \geq n .
$$

Proof. Fix $n$; let $E_{n}$ be an $n$-dimensional subspace of $W_{0}^{1, p}(\Omega)$; we take $u_{n} \in E_{n}$ with norm $\left\|u_{n}\right\|=1$ for $0<\rho<R_{0}$; we have

$$
\begin{aligned}
J\left(\rho u_{n}\right)= & I\left(\rho u_{n}\right) \\
= & \frac{1}{p} \rho^{p}-\frac{1}{p^{*}(t)} \rho^{p^{*}(t)} \\
& \times \int_{\Omega} \frac{\left|u_{n}\right|^{p^{*}(t)}}{|x|^{t}} d x-\frac{\lambda}{q} \rho^{q} \int_{\Omega} \frac{\left|u_{n}\right|^{q}}{|x|^{s}} d x .
\end{aligned}
$$

Since $E_{n}$ is a space of finite dimension, all the norms in $E_{n}$ are equivalent. If we define

$$
\begin{gathered}
\alpha_{n}=\inf \left\{\int_{\Omega} \frac{|u|^{p^{*}(t)}}{|x|^{t}} d x: u \in E_{n},\|u\|=1\right\}>0, \\
\beta_{n}=\inf \left\{\int_{\Omega} \frac{|u|^{q}}{|x|^{s}} d x: u \in E_{n},\|u\|=1\right\}>0,
\end{gathered}
$$

we have

$$
J\left(\rho u_{n}\right) \leq \frac{1}{p} \rho^{p}-\frac{\alpha_{n}}{p^{*}(t)} \rho^{p^{*}(t)}-\frac{\lambda \beta_{n}}{q} \rho^{q},
$$

and we can choose $\varepsilon$ (which depends on $n$ ), and $\eta<R_{0}$, such that $J(\eta u) \leq-\varepsilon$ if $u \in E_{n}$ and $\|u\|=1$.

Let $S_{\eta}=\left\{u \in W_{0}^{1, p}(\Omega):\|u\|=\eta\right\}$. Consider $S_{\eta} \cap E_{n} \subset$ $\left\{u \in W_{0}^{1, p}(\Omega): J(u) \leq-\varepsilon\right\}$; therefore, by Lemma 3, we see that

$$
\gamma\left(\left\{u \in W_{0}^{1, p}(\Omega): J(u) \leq-\varepsilon(n)\right\}\right) \geq \gamma\left(S_{\eta} \cap E_{n}\right)=n .
$$

We are now in a position to prove our main result. 
Proof of Theorem 1. Let $\Sigma_{k}=\left\{C \subset W_{0}^{1, p}(\Omega)-\{0\}\right.$, $C$ is closed, $C=-C, \gamma(C) \geq k\}, c_{k}=\inf _{C \in \Sigma_{k}} \sup _{u \in C} J(u)$, $K_{c}=\left\{u \in W_{0}^{1, p}(\Omega), J^{\prime}(u)=0, J(u)=c\right\}$, and suppose that $0<\lambda<\lambda_{0}$ where $\lambda_{0}$ is the constant given by Lemma 4 . We claim that if $k, r \in N$ are such that $c=c_{k}=c_{k+1}=\cdots=c_{k+r}$, then $\gamma\left(K_{c}\right) \geq r+1$.

In fact, denote $J^{-\varepsilon}=\left\{u \in W_{0}^{1, p}(\Omega): J(u) \leq-\varepsilon\right\}$; by Lemma 5 , we see that for any $k \in N$, there is a $\varepsilon(k)>0$, such that $\gamma\left(J^{-\varepsilon(k)}\right) \geq k$. Since $J$ is continuous and even, $J^{-\varepsilon(k)} \in \Sigma_{n}$ and $c_{k} \leq-\varepsilon(n)<0$. As $J$ is bounded from below, we see that $c_{k}>-\infty$ for all $k \in N$.

Suppose that $c=c_{k}=c_{k+1}=\cdots=c_{k+r}<0$; then $J$ satisfies $(P S)_{c}$ condition by Lemma 2 , and it is easy to see that $K_{c}$ is a compact set.

If $\gamma\left(K_{c}\right) \leq r$, then there is a closed and symmetric set $U$ with $K_{c} \subset U$ and $\gamma(U) \leq r$ by Lemma 3. Since $c<0$, we can also assume that the closed set $U \subset J^{0}$. Since $J$ satisfies $(P S)_{c}$ condition for $c<0$, by the Deformation Lemma, there is an odd homeomorphism,

$$
\eta: W_{0}^{1, p}(\Omega) \longrightarrow W_{0}^{1, p}(\Omega)
$$

such that $\eta\left(J^{c+\delta}-U\right) \subset J^{c-\delta}$ for some $\delta$ with $0<\delta<-c$.

Since $c=c_{k+r}=\inf _{C \in \Sigma_{k}} \sup _{u \in C} J(u)$, there exists an $A \in$ $\Sigma_{k+r}$, such that

$$
\begin{gathered}
\sup _{u \in C} J(u)<c+\delta, \text { i.e., } A \subset J^{c+\delta}, \\
\eta(A-U) \subset \eta\left(J^{c+\delta}-U\right) \subset J^{c-\delta} .
\end{gathered}
$$

But by Lemma 3 and $\gamma(U) \leq r$, we have

$$
\begin{gathered}
\gamma(\overline{A-U}) \geq \gamma(A)-\gamma(U) \geq n, \\
\gamma(\eta \overline{A-U}) \gamma(\overline{A-U}) \geq n .
\end{gathered}
$$

Hence, $\eta \overline{A-U} \in \Sigma_{k}$ and $\sup _{u \in \eta \overline{A-U}} \geq c_{k}=c$, which contradicts to (50). So we have proved that $\gamma\left(K_{c}\right) \geq r+1$.

Now if for all $k \in N$, we have $\Sigma_{k+1} \subset \Sigma_{k}, c_{k} \leq c_{k+1}<0$. If all $c_{k}$ are distinct, then $\gamma\left(K_{c_{k}}\right) \geq 1$, and we see that $\left\{c_{k}\right\}$ is a sequence of distinct critical values of $J$; if for some $k_{0}$, there is a $r \geq 1$ such that

$$
c=c_{k_{0}}=c_{k_{0+1}}=\cdots=c_{k_{0+r}},
$$

then

$$
\gamma\left(K_{c_{k_{0}}}\right) \geq r+1,
$$

which shows that $K_{c_{k_{0}}}$ contains infinitely many distinct elements.

Since $J(u)=I(u)$ if $J(u)<0$, we see that there are infinitely many critical points of $I(u)$. The theorem is proved.

\section{Conflict of Interests}

The authors declare that there is no conflict of interests regarding the publication of this paper.

\section{Acknowledgments}

This research is supported by Ningbo Scientific Research Foundation (2009B21003), K. C. Wong Magna Fund in Ningbo University, NSF of Hebei Province (A2013209278), and National Natural Science Foundation of China (nos. 61271398, 11220248 and 11175092).

\section{References}

[1] L. Caffarelli, R. Kohn, and L. Nirenberg, "First order interpolation inequalities with weights," Compositio Mathematica, vol. 53 , no. 3, pp. 259-275, 1984.

[2] G. H. Hardy, J. E. Littlewood, and G. Pólya, Inequalities, Cambridge Mathematical Library, Cambridge University Press, Cambridge, UK, 1988.

[3] D. Kang, "On the quasilinear elliptic problems with critical Sobolev-Hardy exponents and Hardy terms," Nonlinear Analysis: Theory, Methods \& Applications, vol. 68, no. 7, pp. 1973-1985, 2008.

[4] D. Kang, "On the quasilinear elliptic problem with a critical Hardy-Sobolev exponent and a Hardy term," Nonlinear Analysis: Theory, Methods \& Applications, vol. 69, no. 8, pp. 24322444, 2008.

[5] N. Ghoussoub and C. Yuan, "Multiple solutions for quasi-linear PDEs involving the critical Sobolev and Hardy exponents," Transactions of the American Mathematical Society, vol. 352, no. 12, pp. 5703-5743, 2000.

[6] L. Wang, Q. Wei, and D. Kang, "Multiple positive solutions for $p$-Laplace elliptic equations involving concave-convex nonlinearities and a Hardy-type term," Nonlinear Analysis: Theory, Methods \& Applications, vol. 74, no. 2, pp. 626-638, 2011.

[7] T. S. Hsu, "Multiple positive solutions for a quasilinear elliptic problem involving critical Sobolev-Hardy exponents and concave-convex nonlinearities," Nonlinear Analysis: Theory, Methods \& Applications, vol. 74, no. 12, pp. 3934-3944, 2011.

[8] T. S. Hsu, "Multiple positive solutions for semilinear elliptic equations involving Hardy terms and critical Sobolev-Hardy exponents," Journal of Mathematical Sciences, vol. 3, no. 2, pp. 243-266, 2009.

[9] J. G. Azorero and I. P. Alonso, "Multiplicity of solutions for elliptic problems with critical exponent or with a nonsymmetric term," Transactions of the American Mathematical Society, vol. 323, no. 2, pp. 877-895, 1991.

[10] P. L. Lions, "The concentration-compactness principle in the calculus of variations. The limit case. I," Revista Matemática Iberoamericana, vol. 1, no. 1, pp. 145-201, 1985.

[11] P. L. Lions, "The concentration-compactness principle in the calculus of variations. The limit case. II," Revista Matemática Iberoamericana, vol. 1, no. 2, pp. 45-121, 1985.

[12] P. H. Rabinowitz, Minimax Methods in Critical Point Theory with Applications to Differential Equations, CBMS Regional Conference Series in Mathematics, American Mathematical Society, Providence, RI, USA, 1986. 


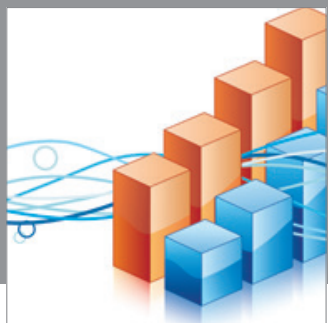

Advances in

Operations Research

mansans

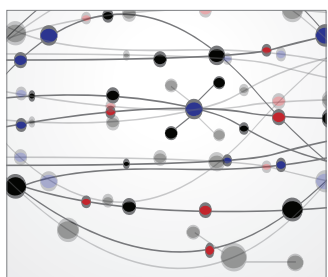

The Scientific World Journal
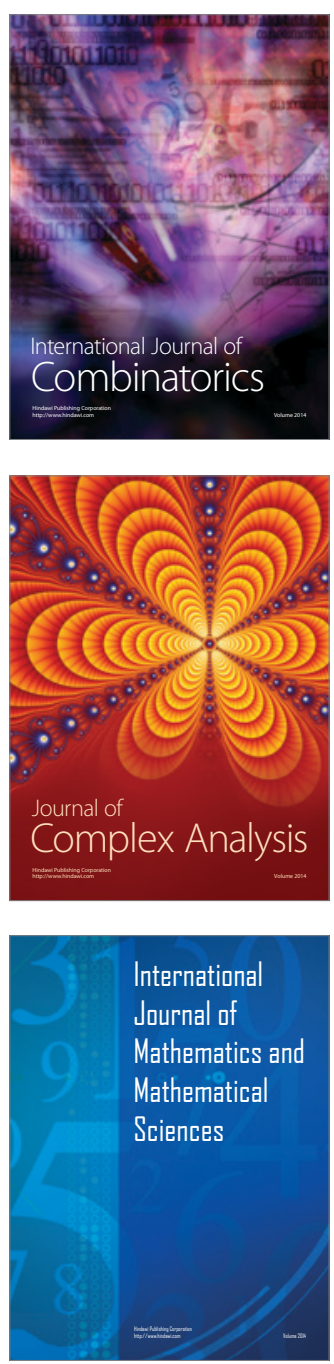
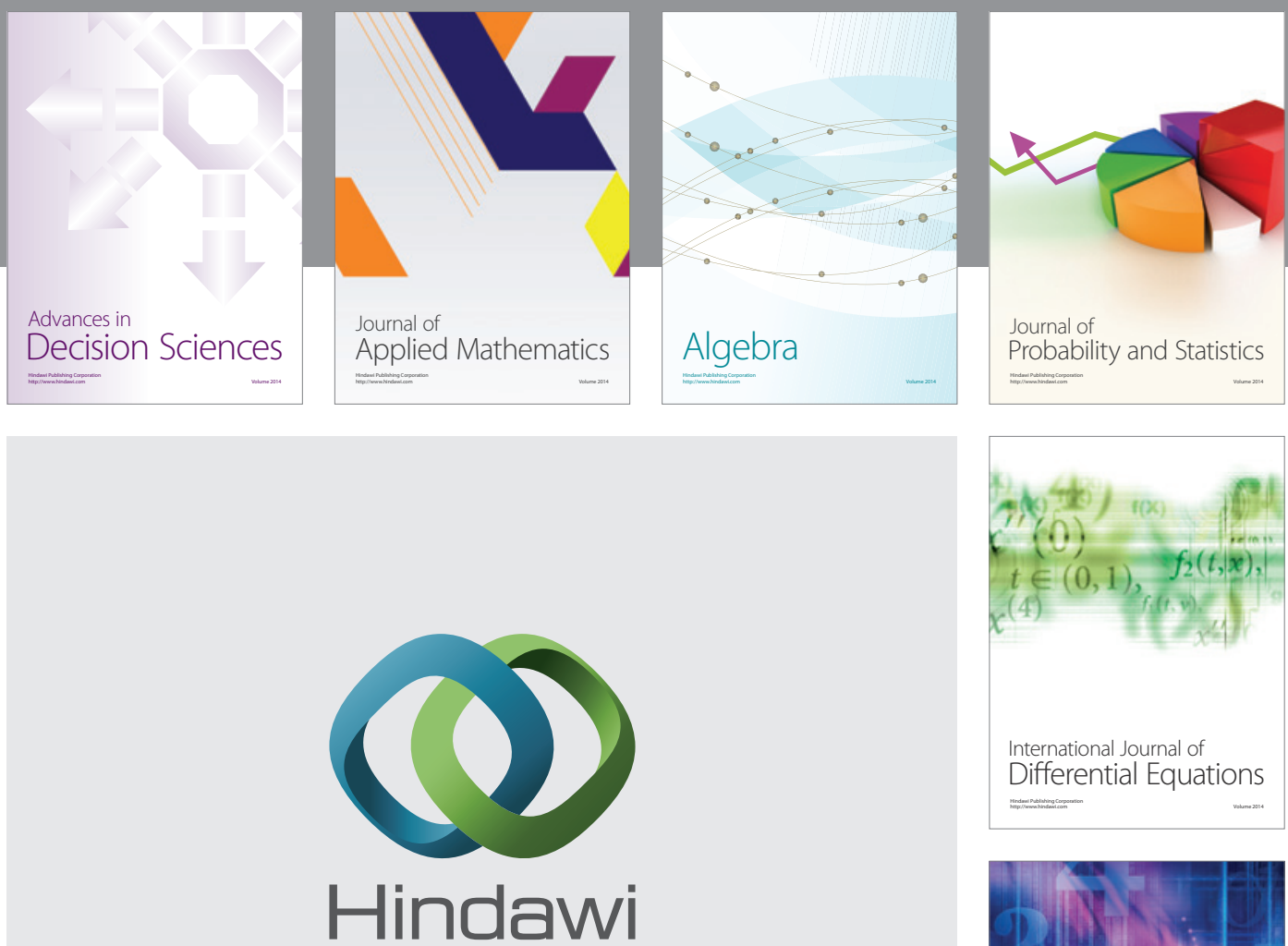

Submit your manuscripts at http://www.hindawi.com
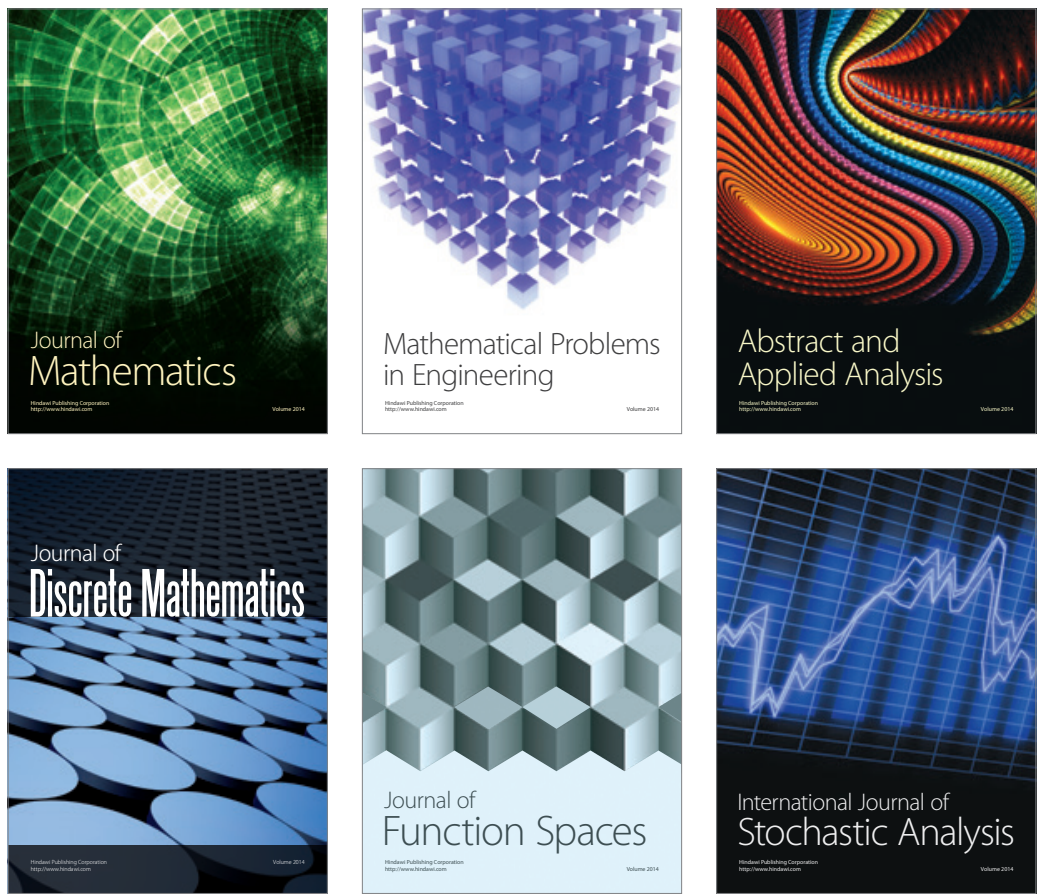

Journal of

Function Spaces

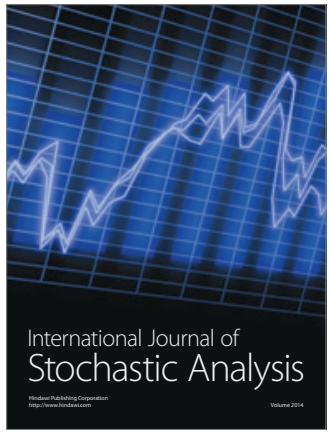

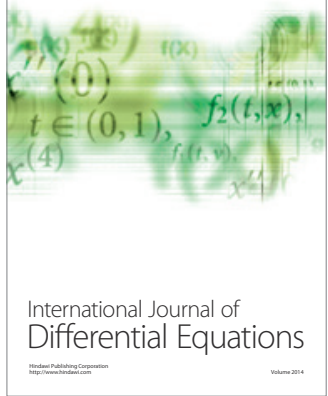
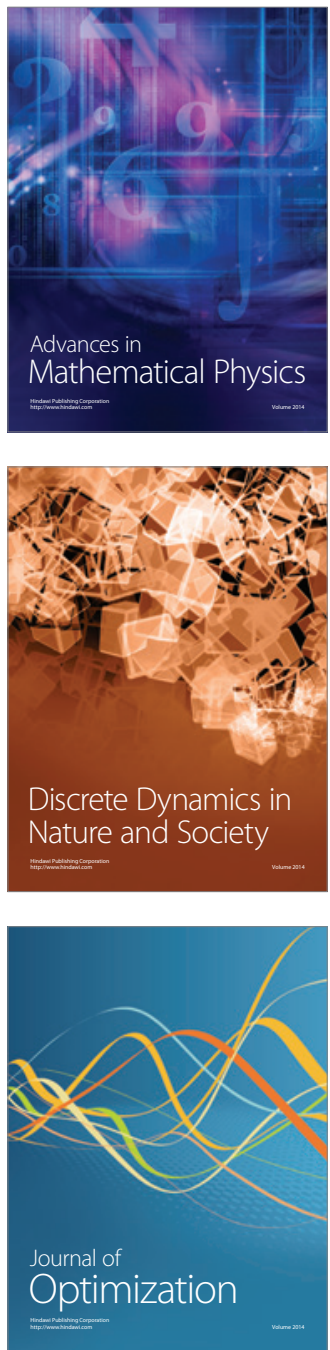